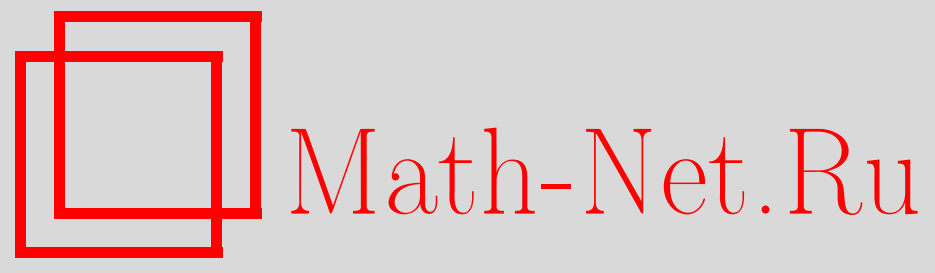

X. А. Хачатрян, А. С. Петросян, О знакопеременных и ограниченных решениях одного класса интегральных уравнений на всей оси с монотонной нелинейностью, Вестн. Сам. гос. техн. ун-та. Сер. Физ.-мат. науки, 2020, номер 4, 644-662

DOI: https://doi.org/10.14498/vsgtu1790

Использование Общероссийского математического портала MathNet.Ru подразумевает, что вы прочитали и согласны с пользовательским соглашением http://www.mathnet.ru/rus/agreement

Параметры загрузки:

IP: 107.22 .136 .117

26 апреля 2023 г., 16:51:42

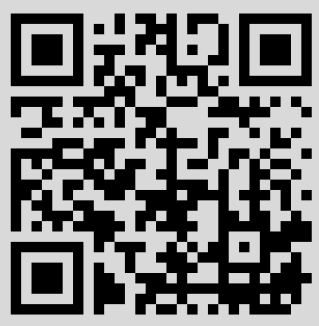


Вестн. Сам. гос. техн. ун-та. Сер. Физ.-мат. науки. 2020. Т. 24, № 4 . С. $644-662$ ISSN: 2310-7081 (online), 1991-8615 (print)

УДК 517.968.74

\section{О знакопеременных и ограниченных решениях одного класса интегральных уравнений на всей оси с монотонной нелинейностью}

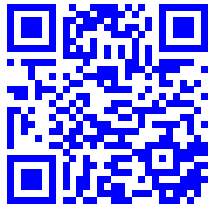

(C) X. A. Хачатрян ${ }^{1,2,3}$, A. С. Петросян ${ }^{1,4}$

1 Московский государственный университет имени М. В. Ломоносова, Россия, 119992, Москва, Ленинские горы, 1.

2 Институт математики НАН Армении, Армения, 0019, Ереван, пр-т Маршала Баграмяна, 24/5.

3 Ереванский государственный университет, Армения, 0025, Ереван, ул. А. Манукяна, 1.

4 Национальный аграрный университет Армении, Армения, 0009, Ереван, ул. Маршала Теряна, 74.

\section{Аннотация}

Работа посвящена исследованию существования, а также анализу качественных свойств решений для одного класса интегральных уравнений с монотонной нелинейностью на всей прямой. Указанный класс уравнений возникает в кинетической теории газов. Доказаны конструктивные теоремы существования ограниченных решений и изучены определенные качественные свойства построенных решений. В конце работы приведены конкретные прикладные примеры указанных уравнений.

Ключевые слова: монотонность, нелинейность, ядро, выпуклость, ограниченное решение.

Получение: 10 июня 2020 г. / Исправление: 16 октября 2020 г.

Принятие: 16 ноября 2020 г. / Публикация онлайн: 19 декабря 2020 г.

\section{Научная статья}

2 (ㅇ)(ㄱ) Контент публикуется на условиях лицензии Creative Commons Attribution 4.0 International (https://creativecommons.org/licenses/by/4.0/deed.ru)

\section{Образец для цитирования}

$\mathrm{Xачатрян} \mathrm{X.} \mathrm{А.,} \mathrm{Петросян} \mathrm{А.} \mathrm{С.} \mathrm{О} \mathrm{знакопеременных} \mathrm{и} \mathrm{ограниченных} \mathrm{решениях}$ одного класса интегральных уравнений на всей оси с монотонной нелинейностью // Вестн. Сам. гос. техн. ун-та. Сер. Физ.-мат. науки, 2020. Т. 24, № 4. С. 644-662. https://doi.org/10.14498/vsgtu1790.

\section{Сведения об авторах}

Хачатур Агавардович Хачатрян (1) https://orcid.org/0000-0002-4835-943X доктор физико-математических наук, профессор; e-mail: khach82@rambler.ru Айкануш Самвеловна Петросян (D) https://orcid.org/0000-0002-7172-4730 кандидат физико-математических наук, доцент; e-mail: haykuhi25@mail.ru 


\section{1. Введение и постановка задачи}

Рассмотрим следующий класс интегральных уравнений на всей прямой

$$
Q(f(x))=\int_{-\infty}^{\infty} K(x, t) f(t) d t, \quad-\infty<x<+\infty,
$$

с монотонной нелинейностью относительно искомой функции $f(x)$. В уравнении (1) ядро $K$ допускает следующее представление:

$$
K(x, t)=\int_{a}^{b} \alpha(x, s) e^{-\alpha(x, s)|x-t|} d \sigma(s) \quad(x, t) \in \mathbb{R} \times \mathbb{R},
$$

где $\sigma(s)$ - монотонно неубывающая функция на $[a, b), 0<a<b \leqslant+\infty$ и удовлетворяет условию:

$$
\int_{a}^{b} d \sigma(s)=\frac{1}{2}
$$

Функция $\alpha(x, s)$ определена на множестве $\mathbb{R} \times[a, b)$ и обладает следующими свойствами:

$\left.\alpha_{1}\right) \alpha \in C(\mathbb{R} \times[a, b))$;

$\left.\alpha_{2}\right)$ существует число $\varepsilon>0$ такое, что $\alpha(x, s) \geqslant \varepsilon>0,(x, s) \in \mathbb{R} \times[a, b)$;

$\left.\alpha_{3}\right) \alpha(x, s)$ симметрична по первому аргументу:

$$
\alpha(-x, s)=\alpha(x, s), x \geqslant 0, s \in[a, b)
$$

$\left.\alpha_{4}\right)$ имеет место неравенство:

$$
\delta:=\sup _{(x, s) \in \mathbb{R}^{+} \times[a, b)}\left(1-\frac{\varepsilon}{\alpha(x, s)}\right)<1 .
$$

Нелинейность $Q$ определена на множестве $\mathbb{R}$ и удовлетворяет следующим условиям (см. рис. 1):

$\left.q_{1}\right)$ существует число $\eta>0$ такое, что на отрезке $[-\eta, \eta]$ функция $Q$ является непрерывной и нечетной;

$\left.q_{2}\right) Q(\eta)=\eta$ и функция $Q$ выпукла вниз на отрезке $[0, \eta]$, причем число $\eta>0$ является первым положительным корнем уравнения $Q(u)=u$;

$\left.q_{3}\right) Q$ монотонно возрастает на отрезке $[-\eta, \eta]$;

$\left.q_{4}\right)$ уравнение $Q(u)=\frac{u}{2}$ имеет положительное решение $\xi<\eta$.

Уравнение (1) имеет непосредственное применение в физической кинетике, а именно в кинетической теории газов (см. [1,2]). Следует отметить, что в линейном случае, когда $Q(u)=u$ и $K(x, t)=0$ при $t<0$, уравнение (1) достаточно подробно изучалось в работе [3]. В том частном случае, когда $Q(u)=u^{p}, p>2$ - нечетное число, а функция $\alpha$ кроме условий $\left.\left.\alpha_{1}\right)-\alpha_{4}\right)$ удовлетворяет также дополнительному условию $\sup _{(x, s) \in \mathbb{R} \times[a, b)} \alpha(x, s)<2 \varepsilon$, в работе [4] были обсуждены вопросы существования ограниченных знакопеременных и нетривиальных решений для уравнения (1).

Небезынтересно также отметить, что соответствующее нелинейное уравнение на полуоси, при существенно иных ограничениях на нелинейность, исследовалось в работе [5]. 
В настоящей работе при более слабых и общих ограничениях на функции $Q$ и $\alpha$ мы докажем конструктивные теоремы существования ограниченных нетривиальных непрерывных и знакопеременных решений, а также исследуем некоторые качественные свойства построенных решений. В конце работы приведем несколько частных и прикладных примеров функций $Q$ и $\alpha$ для иллюстрации полученных результатов.

Решение уравнения (1) будем искать в следующем классе непрерывных на $\mathbb{R}$ функций:

$$
\Pi:=\{\varphi: \varphi(-x)=-\varphi(x), x \geqslant 0, \varphi \in C(\mathbb{R}) \cap M(\mathbb{R})\},
$$

где $M(\mathbb{R})$ - пространство ограниченных функций на множестве $\mathbb{R}$.

\section{2. Обозначения и вспомогательные факты}

\section{1. Сведение уравнения (1) к нелинейному}

\section{интегральному уравнению на полуоси}

Проводя рассуждения, такие же, как в работе [4], решение уравнения (1) можем свести к решению следующего нелинейного интегрального уравнения на положительной полупрямой:

$$
Q(F(x))=\int_{0}^{\infty}\left(K(x, t)-K_{0}(x, t)\right) F(t) d t, \quad x \geqslant 0,
$$

относительно искомой неотрицательной функции $F \in C\left(\mathbb{R}^{+}\right) \cap M\left(\mathbb{R}^{+}\right)$, где ядро $K(x, t)$ задается согласно формулы $(2)$, а $K_{0}(x, t)$ имеет следующий вид:

$$
K_{0}(x, t)=\int_{a}^{b} \alpha(x, s) e^{-\alpha(x, s)(x+t)} d \sigma(s), \quad(x, t) \in \mathbb{R}^{+} \times \mathbb{R}^{+} .
$$

При этом, если функция $F$ является неотрицательным нетривиальным непрерывным и ограниченным на $\mathbb{R}^{+}$решением уравнения $(4)$, то

$$
f(x)= \begin{cases}F(x), & x \geqslant 0 \\ -F(-x), & x<0\end{cases}
$$

будет решением уравнения (1), причем $f \in \Pi$.

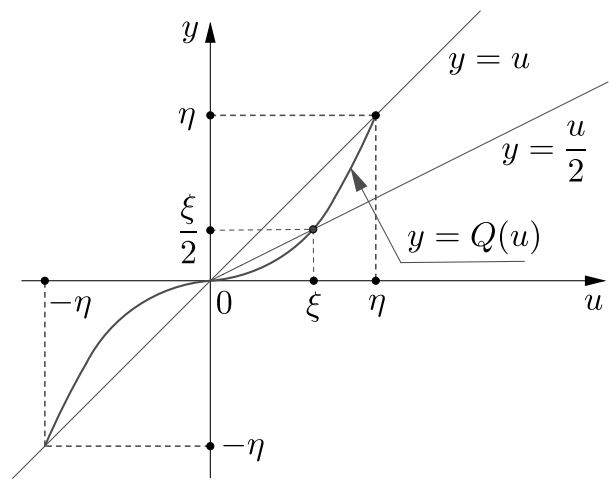

Рис. 1. [Figure 1] 
Более того, если $f \in \Pi$ является нетривиальным решением уравнения (1), то $F(x)=f(x), x \geqslant 0$, будет решением уравнения (4).

Ниже займемся решением уравнения (4). С этой целью мы сначала проведем исследование для специальных вспомогательных линейных уравнений типа Вольтерра.

\section{2. Неоднородное уравнение типа Вольтерра с монотонно убывающим свободным членом}

Рассмотрим следующее неоднородное интегральное уравнение с переменным нижним пределом

$$
\psi(x)=g(x)+\int_{x}^{\infty} v(x, t) \psi(t) d t, \quad x \in \mathbb{R}^{+},
$$

относительно искомой неотрицательной функции $\psi(x)$, где

$$
\begin{gathered}
g(x) \geqslant 0, \quad x \in \mathbb{R}^{+}, \quad g(x) \not \equiv 0, \quad g \in L_{1}\left(\mathbb{R}^{+}\right) \cap M\left(\mathbb{R}^{+}\right), \\
g(x) \text { монотонно убывает по } x \text { на } \mathbb{R}^{+} .
\end{gathered}
$$

Ядро $v(x, t)$ допускает следующее представление через функцию $\alpha(x, s)$ :

$$
v(x, t)=2 K(x, t) \theta(t-x), \quad(x, t) \in \mathbb{R}^{+} \times \mathbb{R}^{+},
$$

где $\theta$ - известная функция Хевисайда.

Уравнение (5) перепишем в операторной форме:

$$
(I-V) \psi=g
$$

где $I$ - единичный оператор, а $V$ - интегральный оператор Вольтерра следующего вида:

$$
(V f)(x)=\int_{x}^{\infty} v(x, t) f(t) d t, \quad x \in \mathbb{R}^{+} .
$$

Легко заметить, что оператор $V$ действует в пространстве $M\left(\mathbb{R}^{+}\right)$. Это сразу следует из свойств функций $\alpha$ и $\sigma$ (см. условия (3) и $\left.\left.\alpha_{1}\right)-\alpha_{4}\right)$ ). Рассмотрим следующую задачу факторизации: для интегрального оператора $V$ найти такой оператор Вольтерра $W$, действующий из $M\left(\mathbb{R}^{+}\right)$в $M\left(\mathbb{R}^{+}\right)$:

$$
(W f)(x)=\int_{x}^{\infty} W(x, t) f(t) d t, \quad f \in M\left(\mathbb{R}^{+}\right),
$$

чтобы имела место факторизация

$$
I-V=(I-W)(I-U)
$$

где

$$
(U f)(x)=\varepsilon \int_{x}^{\infty} e^{-\varepsilon(t-x)} f(t) d t, \quad x \in \mathbb{R}^{+}, \quad f \in M\left(\mathbb{R}^{+}\right) .
$$


Факторизация (9) понимается как равенство операторов, действующих в пространстве $M\left(\mathbb{R}^{+}\right)$. Из результатов работы [6] следует, что ядро оператора $W$ имеет следующий вид:

$$
W(x, t)=2 \int_{a}^{b}(\alpha(x, s)-\varepsilon) e^{-\alpha(x, s)(t-x)} d \sigma(s) \theta(t-x), \quad(x, t) \in \mathbb{R}^{+} \times \mathbb{R}^{+} .
$$

Из свойств $\left.\left.\alpha_{1}\right)-\alpha_{4}\right)$ легко следует, что

$$
\begin{gathered}
W \in C\left(\mathbb{R}^{+} \times \mathbb{R}^{+}\right), \quad W(x, t) \geqslant 0, \quad(x, t) \in \mathbb{R}^{+} \times \mathbb{R}^{+}, \\
\rho:=\sup _{x \in \mathbb{R}^{+}} \int_{0}^{\infty} W(x, t) d t \leqslant 2 \delta \int_{a}^{b} d \sigma(s)=\delta<1 .
\end{gathered}
$$

Из (10) и (11) имеем, что оператор $W$ является сжимающим в пространстве $M\left(\mathbb{R}^{+}\right)$. Используя факторизацию (9), уравнение (5) перепишем в следующем виде:

$$
(I-W)(I-U) \psi=g
$$

Обозначим через

$$
\Phi=(I-U) \psi
$$

Тогда функция $\Phi$ будет удовлетворять следующему неоднородному уравнению типа Вольтерра:

$$
(I-W) \Phi=g
$$

Запишем уравнение (12) в развернутом виде:

$$
\Phi(x)=g(x)+\int_{x}^{\infty} W(x, t) \Phi(t) d t, \quad x \geqslant 0 .
$$

Так как свободный член $g$ удовлетворяет условиям (6), (7), в силу того, что оператор $W$ является сжимающим в пространстве $M\left(\mathbb{R}^{+}\right)$, можем утверждать, что уравнение (13) обладает неотрицательным единственным решением в $M\left(\mathbb{R}^{+}\right)$.

Рассмотрим следующие последовательные приближения:

$$
\begin{gathered}
\Phi_{n+1}(x)=g(x)+\int_{x}^{\infty} W(x, t) \Phi_{n}(t) d t, \quad x \geqslant 0, \\
\Phi_{0}(x)=g(x), \quad n=0,1,2, \ldots
\end{gathered}
$$

Применяя метод математической индукции, можно убедиться, что

$$
\Phi_{n}(x) \uparrow \text { по } n, \quad \Phi_{n} \in M\left(\mathbb{R}^{+}\right), \quad n=0,1,2, \ldots
$$

Ниже докажем, что имеет место следующая оценка:

$$
\Phi_{n}(x) \leqslant \frac{g(x)}{1-\rho}, \quad n=0,1,2, \ldots, \quad x \in \mathbb{R}^{+} .
$$

При $n=0$ данное неравенство сразу следует из определения нулевого приближения в итерациях (14). Предположим, что (16) имеет место для некоторого $n \in \mathbb{N}$. Тогда в силу (7), (10) и (11) из (14) будем иметь 


$$
\begin{aligned}
\Phi_{n+1}(x) \leqslant g(x)+\int_{x}^{\infty} & W(x, t) \frac{g(t)}{1-\rho} d t \leqslant \\
& \leqslant g(x)+\frac{g(x)}{1-\rho} \int_{x}^{\infty} W(x, t) d t \leqslant g(x)+\frac{g(x) \rho}{1-\rho}=\frac{g(x)}{1-\rho} .
\end{aligned}
$$

Из (15) и (16) немедленно следует, что последовательность ограниченных функций $\left\{\Phi_{n}(x)\right\}_{n=0}^{\infty}$ имеет поточечный предел, когда $n \rightarrow \infty$ :

$$
\lim _{n \rightarrow \infty} \Phi_{n}(x)=\Phi(x)
$$

Согласно теореме Б. Леви [7], предельная функция $\Phi$ удовлетворяет уравнению (13). Из (15), (16) в силу (6) и (7) заключаем, что

$$
\begin{gathered}
g(x) \leqslant \Phi(x) \leqslant \frac{g(x)}{1-\rho}, \quad x \in \mathbb{R}^{+}, \\
\Phi \in L_{1}\left(\mathbb{R}^{+}\right) \cap M\left(\mathbb{R}^{+}\right) .
\end{gathered}
$$

Таким образом, в силу единственности решения уравнения (13) в $M\left(\mathbb{R}^{+}\right)$ можем утверждать, что построенное при помощи итераций (14) решение является единственным. Решая уравнение $(I-U) \psi=\Phi$, находим

$$
\psi(x)=\Phi(x)+\varepsilon \int_{x}^{\infty} \Phi(t) d t, \quad x \in \mathbb{R}^{+} .
$$

\section{3. Построение нетривиального решения для однородного уравнения типа Вольтерра}

Рассмотрим следующее однородное линейное интегральное уравнение типа Вольтерра относительно искомой функции $\mathscr{L}(x)$ :

$$
\mathscr{L}(x)=\int_{x}^{\infty}(v(x, t)-\tilde{v}(x, t)) \mathscr{L}(t) d t, \quad x \in \mathbb{R}^{+},
$$

где

$$
\tilde{v}(x, t)=2 \int_{a}^{b} \alpha(x, s) e^{-\alpha(x, s)(x+t)} d \sigma(s), \quad(x, t) \in \mathbb{R}^{+} \times \mathbb{R}^{+},
$$

а ядро $v(x, t)$ задается согласно $(8)$.

Из следующего простого неравенства

$$
|x-t| \leqslant x+t, \quad(x, t) \in \mathbb{R}^{+} \times \mathbb{R}^{+}
$$

с учетом условия $\alpha_{1}$ ) получаем

$$
K(x, t) \geqslant K_{0}(x, t), \quad(x, t) \in \mathbb{R}^{+} \times \mathbb{R}^{+},
$$

откуда, в частности, следует

$$
v(x, t) \geqslant \tilde{v}(x, t), \quad(x, t) \in \mathbb{R}^{+} \times \mathbb{R}^{+} .
$$

Имеет место следующая основная лемма. 
Лемма 1. При условиях (3), $\left.\left.\alpha_{1}\right)-\alpha_{4}\right)$ уравнение (17) обладает неотрииательным, нетривиальным, непрерывным и ограниченным на $\mathbb{R}^{+}$решением $\mathscr{L}(x)$, причем $\lim _{x \rightarrow+\infty} \mathscr{L}(x)=1$ u $1-\mathscr{L} \in L_{1}\left(\mathbb{R}^{+}\right), \mathscr{L}(x) \leqslant 1, x \in \mathbb{R}^{+}$.

Доказательст в о. Наряду с уравнением (17) рассмотрим следующие неоднородные интегральные уравнения типа Вольтерра относительно искомых функций $\psi$ и $\tilde{Q}$ :

$$
\begin{gathered}
\psi_{0}(x)=g_{0}(x)+\int_{x}^{\infty} v(x, t) \psi_{0}(t) d t, \quad x \in \mathbb{R}^{+}, \\
\tilde{Q}(x)=g_{1}(x)+\int_{x}^{\infty}(v(x, t)-\tilde{v}(x, t)) \tilde{Q}(t) d t, \quad x \in \mathbb{R}^{+},
\end{gathered}
$$

где

$$
\begin{gathered}
g_{0}(x):=e^{-2 \varepsilon x}, \quad x \in \mathbb{R}^{+}, \\
g_{1}(x):=2 \int_{a}^{b} e^{-2 \alpha(x, s) x} d \sigma(s), \quad x \in \mathbb{R}^{+} .
\end{gathered}
$$

Очевидно, что функция $g_{0}$ удовлетворяет условиям (6) и (7). Следовательно, в силу полученных в п. 2.1 результатов можно утверждать, что уравнение (21) обладает неотрицательным и ограниченным решением вида

$$
\psi_{0}(x)=\Phi_{0}(x)+\varepsilon \int_{x}^{\infty} \Phi_{0}(t) d t, \quad x \in \mathbb{R}^{+},
$$

где $\Phi_{0}(x)$ является решением уравнения (13) со свободным членом $g(x)=g_{0}(x)$ и удовлетворяет следующему двойному неравенству:

$$
e^{-2 \varepsilon x} \leqslant \Phi_{0}(x) \leqslant \frac{e^{-2 \varepsilon x}}{1-\rho}, \quad x \in \mathbb{R}^{+} .
$$

Так как $g_{0} \in C\left(\mathbb{R}^{+}\right), v \in C\left(\mathbb{R}^{+} \times \mathbb{R}^{+}\right)$, то из $(23),(24)$ и (21) следует

$$
\psi_{0}(x) \in L_{1}\left(\mathbb{R}^{+}\right) \cap C\left(\mathbb{R}^{+}\right) .
$$

Таким образом, уравнение (21) обладает неотрицательным непрерывным суммируемым и ограниченным решением $\psi_{0}(x)$. Более того, из $(23)$ и $(24)$ следует также, что функция $\psi_{0}(x) e^{2 \varepsilon x}$ является ограниченной на $\mathbb{R}^{+}$.

Рассмотрим теперь следующие последовательные приближения для уравнения (22):

$$
\begin{gathered}
\tilde{Q}_{n+1}(x)=g_{1}(x)+\int_{x}^{\infty}(v(x, t)-\tilde{v}(x, t)) \tilde{Q}_{n}(t) d t, \quad x \in \mathbb{R}^{+}, \\
\tilde{Q}_{0}(x)=g_{1}(x), \quad n=0,1,2, \ldots
\end{gathered}
$$

Учитывая неравенство (20), индукцией по $n$ нетрудно убедиться, что

$$
\tilde{Q}_{n}(x) \uparrow \text { по } n, \quad x \in \mathbb{R}^{+} .
$$


В силу того, что $g_{1} \in C\left(\mathbb{R}^{+}\right), v, \tilde{v} \in C\left(\mathbb{R}^{+} \times \mathbb{R}^{+}\right)$, индукцией также можно проверить, что

$$
\tilde{Q}_{n} \in C\left(\mathbb{R}^{+}\right), \quad n=0,1,2, \ldots
$$

Ниже докажем справедливость неравенства

$$
\tilde{Q}_{n}(x) \leqslant \psi_{0}(x), \quad n=0,1,2, \ldots, \quad x \in \mathbb{R}^{+},
$$

где $\psi_{0} \in L_{1}\left(\mathbb{R}^{+}\right) \cap C\left(\mathbb{R}^{+}\right)$- решение уравнения $(21)$, обладающее свойствами (23), (24). При $n=0$ неравенство (28) сразу вытекается из следующей цепочки простых неравенств:

$$
\tilde{Q}_{0}(x)=g_{1}(x) \leqslant 2 \int_{a}^{b} e^{-2 \varepsilon x} d \sigma(s)=e^{-2 \varepsilon x}=g_{0}(x) \leqslant \psi_{0}(x), \quad x \in \mathbb{R}^{+},
$$

ибо $\alpha(x, s) \geqslant \varepsilon>0,(x, s) \in \mathbb{R}^{+} \times[a, b)$ и $\int_{a}^{b} d \sigma(s)=\frac{1}{2}$.

Предположим, что неравенство (28) выполняется при некоторых $n \in \mathbb{N}$. Тогда, учитывая (20), (29), из (25) будем иметь

$$
\begin{aligned}
& \tilde{Q}_{n+1}(x) \leqslant g_{0}(x)+\int_{x}^{\infty}(v(x, t)-\tilde{v}(x, t)) \psi_{0}(t) d t \leqslant \\
& \quad \leqslant g_{0}(x)+\int_{x}^{\infty} v(x, t) \psi_{0}(t) d t=\psi_{0}(x), \quad x \in \mathbb{R}^{+} .
\end{aligned}
$$

Таким образом, в силу (26), (27) и (28) можем утверждать, что последовательность непрерывных функций $\left\{\tilde{Q}_{n}(x)\right\}_{n=0}^{\infty}$ имеет поточечный предел, когда $n \rightarrow \infty: \lim _{n \rightarrow \infty} \tilde{Q}_{n}(x)=\tilde{Q}(x)$, причем согласно теореме Б. Леви предельная функция $\tilde{Q}(x)$ удовлетворяет уравнению $(22)$. Из $(26)$ и $(28)$ следует

$$
2 \int_{a}^{b} e^{-2 \alpha(x, s) x} d \sigma(s) \leqslant \tilde{Q}(x) \leqslant \psi_{0}(x), \quad x \in \mathbb{R}^{+} .
$$

Так как $g_{1} \in C\left(\mathbb{R}^{+}\right), v, \tilde{v} \in C\left(\mathbb{R}^{+} \times \mathbb{R}^{+}\right)$, с учетом (30) можно утверждать, что $\tilde{Q} \in C\left(\mathbb{R}^{+}\right)$.

Итак, согласно теореме Дини получаем, что сходимость последовательности функций $\left\{\tilde{Q}_{n}(x)\right\}_{n=0}^{\infty}$ к $\tilde{Q}(x)$ в каждом компакте из $\mathbb{R}^{+}$равномерна. Из $(30),(23)$ и $(24)$ также следует

$$
\tilde{Q} \in L_{1}\left(\mathbb{R}^{+}\right) \cap M\left(\mathbb{R}^{+}\right)
$$

и более того -

$$
\tilde{Q}(x) e^{2 \varepsilon x} \in M\left(\mathbb{R}^{+}\right) .
$$

Таким образом, уравнение (22) обладает неотрицательным, непрерывным, ограниченным и суммируемым на $\mathbb{R}^{+}$решением, более того, имеет место включение (32). 
Теперь прямой проверкой убедимся, что $\tilde{Q}_{\text {тр }}(x) \equiv 1$ является решением уравнения (22). Действительно, учитывая (3), (8), (18) и теорему Фубини [7], имеем

$$
\begin{aligned}
& g_{1}(x)+\int_{x}^{\infty}(v(x, t)-\tilde{v}(x, t)) d t=2 \int_{a}^{b} e^{-2 \alpha(x, s) x} d \sigma(s)+ \\
& +2 \int_{x}^{\infty} \int_{a}^{b} \alpha(x, s) e^{-\alpha(x, s)(t-x)} d \sigma(s) d t-2 \int_{x}^{\infty} \int_{a}^{b} \alpha(x, s) e^{-\alpha(x, s)(x+t)} d \sigma(s) d t= \\
& =2 \int_{a}^{b} e^{-2 \alpha(x, s) x} d \sigma(s)+2 \int_{a}^{b} d \sigma(s)-2 \int_{a}^{b} \alpha(x, s) \int_{x}^{\infty} e^{-\alpha(x, s)(x+t)} d t d \sigma(s)= \\
& =2 \int_{a}^{b} e^{-2 \alpha(x, s) x} d \sigma(s)+1-2 \int_{a}^{b} e^{-2 \alpha(x, s) x} d \sigma(s)=1 .
\end{aligned}
$$

Индукцией по $n$ легко можно проверить справедливость неравенства

$$
\tilde{Q}_{n}(x) \leqslant 1, \quad n=0,1,2, \ldots, \quad x \in \mathbb{R}^{+} .
$$

Следовательно, $\tilde{Q}(x) \leqslant \tilde{Q}_{\text {тр }}(x), x \in \mathbb{R}^{+}$. Из свойств $(31)$ и $(32)$ следует

$$
\tilde{Q}(x) \not \equiv \tilde{Q}_{\text {тр }}(x) .
$$

В силу линейности уравнения (22) функция

$$
\mathscr{L}(x)=\tilde{Q}_{\text {тр }}(x)-\tilde{Q}(x)=1-\tilde{Q}(x) \geqslant 0
$$

будет удовлетворять однородному уравнению (17). Из свойств функции $\tilde{Q}$ (см. (31), (32)) приходим к завершению доказательства.

\section{3. Разрешимость уравнения (4)}

Рассмотрим следующие последовательные приближения:

$$
\begin{gathered}
Q\left(F_{n+1}(x)\right)=\int_{0}^{\infty}\left(K(x, t)-K_{0}(x, t)\right) F_{n}(t) d t, \quad x \geqslant 0, \\
F_{0}(x)=\eta, \quad n=0,1,2, \ldots
\end{gathered}
$$

Индукцией по $n$ докажем, что

$$
\begin{gathered}
F_{n}(x) \downarrow \text { по } n, \\
F_{n}(x) \geqslant \xi \mathscr{L}(x), \quad n=0,1,2, \ldots, \quad x \in \mathbb{R}^{+}, \\
F_{n} \in C\left(\mathbb{R}^{+}\right), \quad n=0,1,2, \ldots,
\end{gathered}
$$

где $\mathscr{L}(x)$ - решение уравнения $(17)$ (см. лемму 1).

Сперва убедимся, что $0 \leqslant F_{1}(x) \leqslant F_{0}(x), x \in \mathbb{R}^{+}$. Учитывая (19), (3), из (33) будем иметь 


$$
\begin{gathered}
Q\left(F_{1}(x)\right) \leqslant \eta \int_{0}^{\infty} K(x, t) d t=\eta \int_{a}^{b} \int_{0}^{\infty} \alpha(x, s) e^{-\alpha(x, s)|x-t|} d t d \sigma(s) \leqslant \\
\leqslant \eta \int_{a}^{b} \alpha(x, s) \int_{-\infty}^{\infty} e^{-\alpha(x, s)|y|} d y d \sigma(s)=2 \eta \int_{a}^{b} d \sigma(s)=\eta=Q(\eta), \\
Q\left(F_{1}(x)\right) \geqslant 0=Q(0), \quad x \in \mathbb{R}^{+} .
\end{gathered}
$$

Из (37) и (38) в силу монотонности функции $Q$ следует справедливость неравенств

$$
0 \leqslant F_{1}(x) \leqslant F_{0}(x), \quad x \in \mathbb{R}^{+}
$$

Неравенство (35) для $n=0$ сразу следует из оценки

$$
F_{0}(x)=\eta>\xi \geqslant \xi \mathscr{L}(x), \quad x \in \mathbb{R}^{+}
$$

Непрерывность нулевого приближения в итерациях (33) очевидна. Предположим, что для некоторого натурального $n$ имеет место неравенство

$$
0 \leqslant F_{n}(x) \leqslant F_{n-1}(x), \quad x \in \mathbb{R}^{+} .
$$

Тогда, вновь используя (19), из (33) получим

$$
\begin{gathered}
Q\left(F_{n+1}(x)\right) \leqslant \int_{0}^{\infty}\left(K(x, t)-K_{0}(x, t)\right) F_{n-1}(t) d t=Q\left(F_{n}(x)\right), \quad x \in \mathbb{R}^{+} \\
Q\left(F_{n+1}(x)\right) \geqslant 0=Q(0), \quad x \in \mathbb{R}^{+}
\end{gathered}
$$

Снова используя монотонность функции $Q$, из (39) и (40) будем иметь

$$
0 \leqslant F_{n+1}(x) \leqslant F_{n}(x), \quad x \in \mathbb{R}^{+} .
$$

Предположим теперь, что $F_{n}(x) \geqslant \xi \mathscr{L}(x), x \in \mathbb{R}^{+}$, для некоторого $n \in \mathbb{N}$. Тогда, учитывая (19) и условия $\left.\left.q_{1}\right)-q_{4}\right)$, из (33) получаем

$$
\begin{gathered}
Q\left(F_{n+1}(x)\right) \geqslant \xi \int_{0}^{\infty}\left(K(x, t)-K_{0}(x, t)\right) \mathscr{L}(t) d t \geqslant \\
\geqslant \xi \int_{x}^{\infty}\left(K(x, t)-K_{0}(x, t)\right) \mathscr{L}(t) d t= \\
=\frac{\xi}{2} \int_{x}^{\infty}(v(x, t)-\tilde{v}(x, t)) \mathscr{L}(t) d t=\frac{\xi \mathscr{L}(x)}{2} \geqslant Q(\xi \mathscr{L}(x)), \quad x \in \mathbb{R}^{+},
\end{gathered}
$$

ибо $Q(u) \leqslant \frac{u}{2}, u \in[0, \xi]$ и $0 \leqslant \mathscr{L}(x) \leqslant 1, x \in \mathbb{R}^{+}$.

Наконец, предположим, что $F_{n} \in C\left(\mathbb{R}^{+}\right)$при некотором $n \in \mathbb{N}$. Тогда в силу того, что $K, K_{0} \in C\left(\mathbb{R}^{+} \times \mathbb{R}^{+}\right)$, получаем, что $Q\left(F_{n+1}(x)\right)$ является непрерывной функцией на $\mathbb{R}^{+}$. Так как $0 \leqslant F_{n+1}(x) \leqslant \eta, x \in \mathbb{R}^{+}$и $Q \in C[-\eta, \eta]$, $Q \uparrow$ на $[-\eta, \eta]$, из непрерывности функции $Q\left(F_{n+1}(x)\right)$ следует непрерывность функции $F_{n+1}(x)$ на $\mathbb{R}^{+}$. 
Таким образом, утверждения (34)-(36) полностью доказаны. Следовательно, последовательность непрерывных функций $\left\{F_{n}(x)\right\}_{n=0}^{\infty}$ имеет поточечный предел, когда $n \rightarrow \infty: \lim _{n \rightarrow \infty} F_{n}(x)=F(x)$, причем предельная функция $F$ удовлетворяет уравнению $(4)$ и имеет место следующая двойная оценка:

$$
\xi \mathscr{L}(x) \leqslant F(x) \leqslant \eta, \quad x \in \mathbb{R}^{+},
$$

и в силу непрерывности ядер $K, K_{0}$ и нелинейности $Q$ функция $F \in C\left(\mathbb{R}^{+}\right)$.

Итак, на основе вышеизложенного приходим к следующему результату.

Лемма 2. При условиях (3), $\left.\left.\alpha_{1}\right)-\alpha_{4}\right)$ и $\left.q_{1}\right)-q_{4}$ ) уравнение (4) обладает неотрицательным, нетривиальным, непрерывным и ограниченным на $\mathbb{R}^{+}$решением. Более того, имеет место двойная оценка (41).

\section{4. Асимптотическое поведение решения при дополнительных ограничениях на $\alpha(x, s)$ и $Q(u)$}

\section{1. Ключевая лемма}

Ниже при дополнительных ограничениях на функции $\alpha(x, s)$ и $Q(u)$ мы докажем справедливость следующих соотношений:

1) $\lim _{x \rightarrow+\infty} F(x)=\eta$

2) $\eta-F \in L_{1}\left(\mathbb{R}^{+}\right)$.

Кроме условий $\left.\left.\alpha_{1}\right)-\alpha_{4}\right)$ и $\left.q_{1}\right)-q_{4}$ ), здесь мы предполагаем, что

$$
\begin{gathered}
\alpha_{0}:=\sup _{(x, s) \in \mathbb{R} \times[a, b)} \alpha(x, s) \leqslant 2 \varepsilon, \\
\eta<\frac{3}{2} \xi .
\end{gathered}
$$

Имеет место следующая лемма.

Лемма 3. При условиях (3), $\left.\left.\left.\left.\alpha_{1}\right)-\alpha_{4}\right), q_{1}\right)-q_{4}\right)$ u (42), (43) любое решение уравнения (4), удовлетворяющее неравенством (41), обладает свойствами 1) и 2).

Доказ ательств о. Сперва заметим, что уравнение (4) можно представить в следующем виде:

$$
\begin{aligned}
\eta-Q(F(x))= & \eta \int_{0}^{\infty} K_{0}(x, t) d t+ \\
& +\int_{0}^{\infty} K_{0}(x, t) F(t) d t+\int_{0}^{\infty} K(x, t)(\eta-F(t)) d t, \quad x \geqslant 0,
\end{aligned}
$$

поскольку, согласно теореме Фубини,

$$
\begin{aligned}
& \eta \int_{0}^{\infty}\left(K(x, t) d t+K_{0}(x, t)\right) d t= \\
& =\eta \int_{a}^{b} \int_{0}^{\infty} \alpha(x, s) e^{-\alpha(x, s)|x-t|} d t d \sigma(s)+\eta \int_{a}^{b} \int_{0}^{\infty} \alpha(x, s) e^{-\alpha(x, s)(x+t)} d t d \sigma(s)=
\end{aligned}
$$




$$
\begin{array}{r}
=\eta \int_{a}^{b} \int_{-\infty}^{x} \alpha(x, s) e^{-\alpha(x, s)|y|} d y d \sigma(s)+\eta \int_{a}^{b} \int_{x}^{\infty} \alpha(x, s) e^{-\alpha(x, s)|y|} d y d \sigma(s) \\
=2 \eta \int_{a}^{b} d \sigma(s)=\eta
\end{array}
$$

Так как $\varepsilon \leqslant \alpha(x, s) \leqslant 2 \varepsilon$ (см. условия $\left.\alpha_{2}\right)$ и $\left.(42)\right)$, то с учетом $\left.\left.q_{1}\right)-q_{3}\right),(41)$, (3) и (44) имеем

$$
\begin{aligned}
0 \leqslant \eta-Q(F(x)) \leqslant 2 \eta \frac{\alpha_{0}}{\varepsilon} & \int_{a}^{b} \int_{x}^{\infty} e^{-\varepsilon y} d y d \sigma(s)+\int_{0}^{\infty} K(x, t)(\eta-F(t)) d t= \\
& =\eta \frac{\alpha_{0}}{\varepsilon} e^{-\varepsilon x}+\int_{0}^{\infty} K(x, t)(\eta-F(t)) d t, \quad x \geqslant 0 .
\end{aligned}
$$

Рассмотрим теперь функцию

$$
\chi(u):=\frac{\eta-Q(u)}{\eta-u}, \quad u \in[0, \eta) .
$$

Из свойств $\left.\left.q_{1}\right)-q_{4}\right),(43)$ сразу следует

$$
\begin{gathered}
\chi \in C[0, \eta), \\
\chi(0)=1, \quad \chi(\xi)=\frac{\eta-\frac{\xi}{2}}{\eta-\xi}>2 .
\end{gathered}
$$

В силу выпуклости и монотонности функции $Q$ на интервале $[0, \eta)$ функция $\chi(u)$ будет монотонно возрастающей на $[0, \eta)$ (см. рис. 2).

Следовательно, существует $\xi_{0} \in(0, \xi)$ такое, что

$$
\chi\left(\xi_{0}\right)>2 \text {. }
$$

Зафиксируем число $\xi_{0}$. Так как $\lim _{x \rightarrow+\infty} \mathscr{L}(x)=1$ и $\mathscr{L}(x) \leqslant 1, x \in \mathbb{R}^{+}$, существует число $r>0$ такое, что при $x \geqslant r$ имеет место неравенство

$$
1-\mathscr{L}(x)=|1-\mathscr{L}(x)| \leqslant \frac{\xi-\xi_{0}}{\xi},
$$

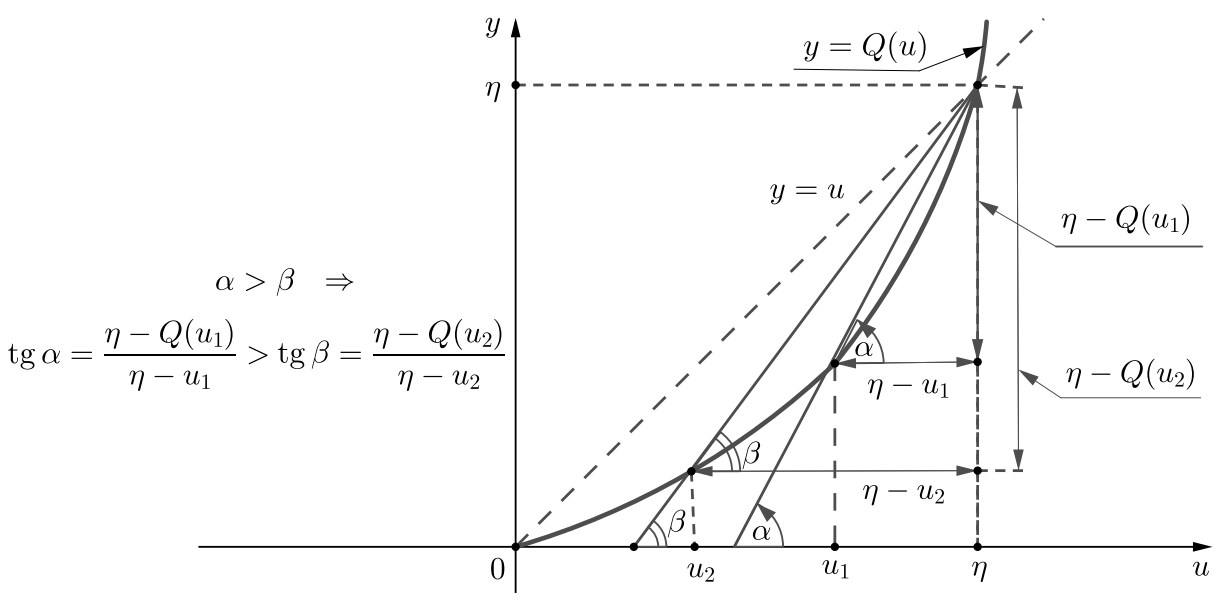

Рис. 2. [Figure 2] 
из которого следует

$$
\mathscr{L}(x) \geqslant \frac{\xi_{0}}{\xi} \text { при } x \geqslant r .
$$

Таким образом, учитывая (41) и (47), можем утверждать, что

$$
F(x) \geqslant \xi_{0} \text { при } x \geqslant r .
$$

Проведем теперь прямую, проходящую через точки $\left(\xi_{0}, Q\left(\xi_{0}\right)\right)$ и $(\eta, \eta)$. Уравнение этой прямой имеет вид (см. рис. 3)

$$
y=\frac{\eta-Q\left(\xi_{0}\right)}{\eta-\xi_{0}} u+\eta \frac{Q\left(\xi_{0}\right)-\xi_{0}}{\eta-\xi_{0}} .
$$

В силу выпуклости функции $Q$ на отрезке $[0, \eta]$ можем утверждать, что при $x \geqslant r$

$$
Q(F(x)) \leqslant \frac{\eta-Q\left(\xi_{0}\right)}{\eta-\xi_{0}} F(x)+\eta \frac{Q\left(\xi_{0}\right)-\xi_{0}}{\eta-\xi_{0}},
$$

из чего следует справедливость неравенства

$$
\eta-Q(F(x)) \geqslant \chi\left(\xi_{0}\right)(\eta-F(x)) \text { при } x \geqslant r .
$$

Так как $K, K_{0} \in C\left(\mathbb{R}^{+} \times \mathbb{R}^{+}\right), Q \in C[0, \eta]$, из (4) в силу (41) и $\left.q_{3}\right)$ получаем $F \in C\left(\mathbb{R}^{+}\right)$. Поскольку $F \in C\left(\mathbb{R}^{+}\right)$, очевидно, что $\eta-F \in L_{1}(0, r)$.

Докажем теперь, что $\eta-F \in L_{1}(r,+\infty)$. Пусть $R>r$ - произвольное конечное число. Проинтегрируем обе части (45) по $x$ в пределах от $r$ до $R$ :

$$
\begin{array}{r}
\int_{r}^{R}(\eta-Q(F(x))) d x \leqslant \frac{\eta \alpha_{0}}{\varepsilon} \int_{r}^{R} e^{-\varepsilon x} d x+\int_{r}^{R} \int_{0}^{\infty} K(x, t)(\eta-F(t)) d t d x \leqslant \\
\leqslant \frac{\eta \alpha_{0}}{\varepsilon^{2}}+\int_{r}^{R} \int_{0}^{r} K(x, t)(\eta-F(t)) d t d x+\int_{r}^{R} \int_{r}^{R} K(x, t)(\eta-F(t)) d t d x+ \\
+\int_{r}^{R} \int_{R}^{\infty} K(x, t)(\eta-F(t)) d t d x
\end{array}
$$

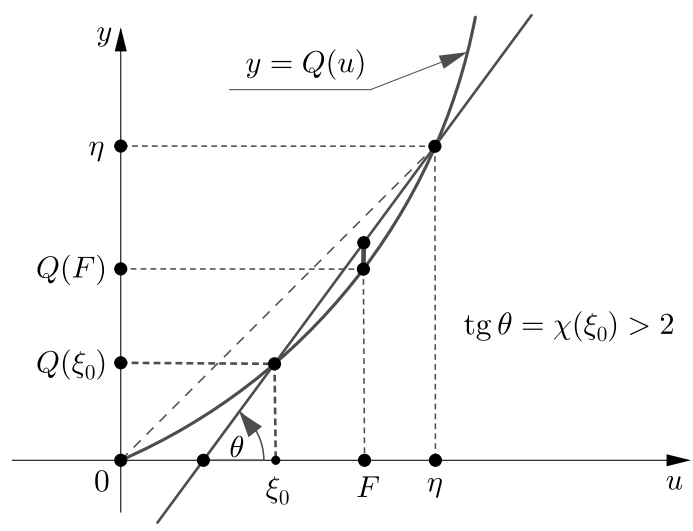

Рис. 3. [Figure 3] 
Заметим, что в силу теоремы Фубини

$$
\begin{gathered}
\int_{r}^{R} \int_{0}^{r} K(x, t) d t d x \leqslant \alpha_{0} \int_{r}^{R} \int_{a}^{b} \int_{0}^{r} e^{-\varepsilon|x-t|} d t d \sigma(s) d x= \\
=\frac{\alpha_{0}}{2} \int_{r}^{R} \int_{x-r}^{x} e^{-\varepsilon|y|} d y d x=\frac{\alpha_{0}}{2} \int_{r}^{R}\left(\int_{x-r}^{\infty} e^{-\varepsilon y} d y-\int_{x}^{\infty} e^{-\varepsilon y} d y\right) d x \leqslant \\
\leqslant \frac{\alpha_{0}}{2 \varepsilon} \int_{r}^{R} e^{-\varepsilon(x-r)} d x \leqslant \frac{\alpha_{0}}{2 \varepsilon} \int_{r}^{\infty} e^{-\varepsilon t} d t=\frac{\alpha_{0}}{2 \varepsilon^{2}} e^{-\varepsilon r} \leqslant \frac{\alpha_{0}}{2 \varepsilon^{2}}<+\infty \\
\int_{r}^{R} \int_{R}^{\infty} K(x, t) d t d x \leqslant \frac{\alpha_{0}}{2} \int_{r}^{R} \int_{R}^{\infty} e^{-\varepsilon|x-t|} d t d x=\frac{\alpha_{0}}{2} \int_{r}^{R} \int_{-\infty}^{x-R} e^{-\varepsilon|y|} d y d x \leqslant \\
\leqslant \frac{\alpha_{0}}{2} \int_{0}^{R} \int_{-\infty}^{x-R} e^{\varepsilon y} d y d x=\frac{\alpha_{0}}{2 \varepsilon} \int_{0}^{R} e^{\varepsilon(x-R)} d x \leqslant \frac{\alpha_{0}}{2 \varepsilon^{2}}<+\infty .
\end{gathered}
$$

В конечном итоге имеем

$$
\int_{r}^{R} \int_{0}^{r} K(x, t) d t d x \leqslant \frac{\alpha_{0}}{2 \varepsilon^{2}}, \quad \int_{r}^{R} \int_{R}^{\infty} K(x, t) d t d x \leqslant \frac{\alpha_{0}}{2 \varepsilon^{2}} .
$$

Учитывая (50) и (41), из (49) получим

$$
\begin{aligned}
\int_{r}^{R}(\eta-Q(F(x))) d x \leqslant \\
\leqslant \frac{\eta \alpha_{0}}{\varepsilon^{2}}+\frac{\eta \alpha_{0}}{2 \varepsilon^{2}}+\frac{\eta \alpha_{0}}{2 \varepsilon^{2}}+\int_{r}^{R} \int_{r}^{R} K(x, t)(\eta-F(t)) d t d x= \\
=\frac{2 \eta \alpha_{0}}{\varepsilon^{2}}+\int_{r}^{R} \int_{r}^{R} K(x, t)(\eta-F(t)) d t d x .
\end{aligned}
$$

Теперь, используя неравенство (48) с учетом (3), (42), $\left.\alpha_{1}\right)$ и теорему Фубини, из (51) будем иметь

$$
\begin{aligned}
& 0 \leqslant \chi\left(\xi_{0}\right) \int_{r}^{R}(\eta-F(x)) d x \leqslant \\
& \leqslant \frac{2 \eta \alpha_{0}}{\varepsilon^{2}}+\int_{r}^{R}(\eta-F(t)) \int_{0}^{\infty} K(x, t) d x d t \leqslant \\
& \quad \leqslant \frac{2 \eta \alpha_{0}}{\varepsilon^{2}}+2 \int_{r}^{R}(\eta-F(t)) d t
\end{aligned}
$$

или в силу (46)-

$$
0 \leqslant \int_{r}^{R}(\eta-F(x)) d x \leqslant \frac{2 \eta \alpha_{0}}{\left(\chi\left(\xi_{0}\right)-2\right) \varepsilon^{2}}
$$


Устремляя число $R$ к бесконечности в (52), получим $\eta-F \in L_{1}(r,+\infty)$ и

$$
0 \leqslant \int_{r}^{\infty}(\eta-F(x)) d x \leqslant \frac{2 \eta \alpha_{0}}{\left(\chi\left(\xi_{0}\right)-2\right) \varepsilon^{2}} .
$$

Окончательно в силу (41) и (53) имеем

$$
\eta-F \in L_{1}\left(\mathbb{R}^{+}\right) \cap M\left(\mathbb{R}^{+}\right) .
$$

Из (45) с учетом (3), $\left.\alpha_{1}\right)$ и (42) следует

$$
0 \leqslant \eta-Q(F(x)) \leqslant \frac{\eta \alpha_{0}}{\varepsilon} e^{-\varepsilon x}+\varepsilon \int_{0}^{\infty} e^{-\varepsilon|x-t|}(\eta-F(t)) d t, \quad x \in \mathbb{R}^{+} .
$$

Так как $\eta-F \in L_{1}\left(\mathbb{R}^{+}\right) \cap M\left(\mathbb{R}^{+}\right), e^{-\varepsilon|x|} \in L_{1}(\mathbb{R}) \cap M(\mathbb{R})$, в силу известного предельного соотношения в операции свертки [8] имеем

$$
\lim _{x \rightarrow+\infty} \int_{0}^{\infty} e^{-\varepsilon|x-t|}(\eta-F(t)) d t=0
$$

Из (54) и (55) сразу получаем

$$
\lim _{x \rightarrow+\infty} Q(F(x))=\eta
$$

Учитывая $\left.\left.q_{1}\right)-q_{3}\right)$, из (56) окончательно получим

$$
\lim _{x \rightarrow+\infty} F(x)=\eta \text {. }
$$

\section{2. Формулировка основных результатов}

Вернемся теперь к уравнению (1). Учитывая формулу

$$
f(x)=\left\{\begin{array}{cc}
F(x), & x \geqslant 0 \\
-F(-x), & x<0
\end{array}\right.
$$

и утверждения леммы 2 и леммы 3, приходим к следующим теоремам.

Теорема 1. При условиях леммы 2 уравнение (1) обладает нетривиальным знакопеременным непрерывным нечетным и ограниченным решением $f(x)$. Более того, данное решение удовлетворяет следующим неравенствам:

$$
\begin{array}{r}
\xi \mathscr{L}(x) \leqslant f(x) \leqslant \eta, \text { при } x \geqslant 0, \\
-\eta \leqslant f(x) \leqslant-\xi \mathscr{L}(-x), \text { nри } x<0 .
\end{array}
$$

Теорема 2. При условиях леммъ 3 любое решение $f(x) \in \Pi$ уравнения (1), удовлетворяющее неравенству (57), обладает следующими предельными и асимптотическими свойствами:

$$
\lim _{x \rightarrow \pm \infty} f(x)= \pm \eta, \quad \eta \pm f \in L_{1}\left(\mathbb{R}^{\mp}\right),
$$

где $\mathbb{R}^{+}:=[0,+\infty), \mathbb{R}^{-}:=(-\infty, 0]$. 


\section{5. Вопрос единственности решения. Примеры}

\section{1. О единственности решения}

Возникает естественный вопрос: будет ли построенное нами решение единственным? Ответ на этот вопрос в общем случае отрицательный. Ниже убедимся, что, например, когда функция $\alpha(x, s)$ является периодической по переменной $x$ с основным периодом $T>0$ (т. е. $\alpha(x+T, s)=\alpha(x, s), x \in \mathbb{R}$, $s \in[a, b))$, то единственность нарушается. Заметим, что тогда уравнение (1), кроме решения $f(x)$, обладает также однопараметрическим семейством ре-

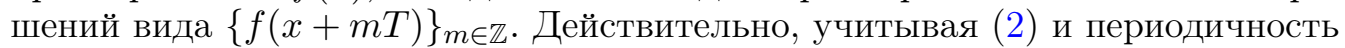
функции $\alpha$, из (1) будем иметь

$$
\begin{gathered}
\int_{-\infty}^{\infty} K(x, t) f(t+m T) d t=\int_{a}^{b} \int_{-\infty}^{\infty} \alpha(x, s) e^{-\alpha(x, s)|x-t|} f(t+m T) d t d \sigma(s)= \\
=\int_{a}^{b} \int_{-\infty}^{\infty} \alpha(x, s) e^{-\alpha(x, s)|x+m T-y|} f(y) d y d \sigma(s)= \\
=\int_{a}^{b} \int_{-\infty}^{\infty} \alpha(x+m T, s) e^{-\alpha(x+m T, s)|x+m T-y|} f(y) d y d \sigma(s)= \\
=Q(f(x+m T)) .
\end{gathered}
$$

\section{2. Примеры функций $Q$ и $\alpha$}

Приведем несколько примеров функций $Q$ и $\alpha$, удовлетворяющих условиям доказанных теорем.

ПРИМЕРЫ ФУНКцИИ $Q$ :

$\left.Q^{a}\right) Q(u)=u^{p}, u \in \mathbb{R}, p>2$ - произвольное нечетное число.

$\left.Q^{b}\right) Q(u)=a u^{p}+(1-a) u, a \in\left(\frac{1}{2}, 1\right], p>2$ - нечетное число.

Подробно остановимся на примере $Q^{b}$. Проверим условия $\left.\left.q_{1}\right)-q_{4}\right)$.

Действительно, легко заметить, что для этой функции положительной неподвижной точкой служит число $\eta=1$, а $\xi=\sqrt{\frac{a-1 / 2}{a}}<1$. Очевидно, что $Q \uparrow$ на $\mathbb{R}, Q \in C(\mathbb{R})$ и $Q(-u)=-Q(u), u \geqslant 0$, причем $Q$ выпукла вниз на отрезке $[0,1]$.

Заметим, что при $a \in\left(\frac{9}{10}, 1\right]$ имеет место также условие $\eta<\frac{3}{2} \xi$. В справедливости последнего неравенства можно убедиться прямой проверкой.

ПРИМЕРЫ ФУНКциИ $\alpha$ :

$\left.\alpha^{a}\right) \alpha(x, s)=\varepsilon+G_{0}(s) e^{-|x|}, x \in \mathbb{R}$, где $G_{0} \in C[a, b)$ и $0 \leqslant G_{0}(s) \leqslant \varepsilon$, $s \in[a, b), \varepsilon>0$.

$\left.\alpha^{b}\right) \alpha(x, s)=\varepsilon+G_{0}(s) \sin ^{2} x, x \in \mathbb{R}$, где $G_{0}$ - удовлетворяет условиям примера $\alpha^{a}$.

Следует отметить, что в случае примера $\alpha^{b}$ (период $T=\pi$ ) уравнение (1) обладает однопараметрическим семейством решений вида $\{f(x+\pi m)\}_{m \in \mathbb{Z}}$.

Конкурирующие интересы. Заявляем, что в отношении авторства и публикации этой статьи конфликта интересов не имеем.

Авторский вклад и ответственность. Все авторы принимали участие в разработке концепции статьи и в написании рукописи. Авторы несут полную ответственность за предоставление окончательной рукописи в печать. Окончательная версия рукописи была одобрена всеми авторами. 
Финансирование. Исследование выполнено за счет гранта Российского научного фонда (проект № 19-11-00223).

\section{Библиографический список}

1. Коган М. Н. Динамика разреженного газа. М.: Наука, 1967. 440 с.

2. Енгибарян Н. Б., Хачатрян А. Х. О некоторых интегральных уравнениях типа свертки в кинетической теории // Ж. вычисл. матем. и матем. физ., 1998. Т. 38, № 3. C. $466-482$.

3. Хачатрян Х. А. Разрешимость консервативного интегрального уравнения на полуоси // Изв. НАН Армении. Математика, 2002. Т. 37, № 4. С. 73-80.

4. Хачатрян Х. А., Сисакян А. А. О разрешимости одного класса нелинейных интегральных уравнений на всей прямой // Вестн. РАУ. Сер. физ.-мат. науки, 2017. № 2. C. $25-40$.

5. Хачатрян А. Х., Хачатрян Х. А. Об одном нелинейном интегральном уравнении типа уравнения Гаммерштейна с некомпактным оператором // Maтем. сб., 2010. Т. 201, № 4. C. 125-136. https://doi.org/10.4213/sm7310.

6. Хачатрян Х. А., Григорян С. А. О нетривиальной разрешимости одного нелинейного интегрального уравнения типа Гаммерштейна-Вольтерра // Владикавк. матем. журн., 2012. Т. 14, № 2. С. 57-66.

7. Колмогоров А. Н., Фомин В. С. Элементы теории функиици и функиионального анализа. М.: Наука, 1976. 543 с.

8. Арабаджян Л. Г., Хачатрян А. С. Об одном классе интегральных уравнений типа свертки // Матем. сб., 2007. Т. 198, №7. С. 45-62. https://doi.org/10.4213/sm1483. 


\title{
MSC: 45G05
}

\section{On alternating and bounded solutions of one class of integral equations on the entire axis with monotonic nonlinearity}

(C) Kh. A. Khachatryan ${ }^{1,2,3}$, H. S. Petrosyan ${ }^{1,4}$

1 Lomonosov Moscow State University, 1, Leninskie Gory, Moscow, 119991, Russian Federation.

2 Institute of Mathematics, National Academy of Sciences of Armenia, 24/5, Marshal Baghramyan pr., Yerevan, 0019, Armenia.

3 Yerevan State University, 1, A. Manukyan str., Yerevan, 0025, Armenia.

4 Armenian National Agrarian University, 74, Marshal Teryan str., Yerevan, 0009, Armenia.

\begin{abstract}
The paper is devoted to the study of the existence and analysis of the qualitative properties of solutions for one class of integral equations with monotonic nonlinearity on the entire line. The indicated class of equations arises in the kinetic theory of gases. The constructive theorems of the existence of bounded solutions are proved, and certain qualitative properties of the constructed solutions are studied. At the end of the paper, specific applied examples of these equations are given.
\end{abstract}

Keywords: monotonicity, nonlinearity, kernel, convexity, limited solution.

Received: $10^{\text {th }}$ June, $2020 /$ Revised: $16^{\text {th }}$ October, $2020 /$ Accepted: $16^{\text {th }}$ November, $2020 /$ First online: $19^{\text {th }}$ December, 2020

Competing interests. We declare that we have no conflicts of interest in the authorship and publication of this article.

Author's Responsibilities. We take full responsibility for submitting the final manuscript in print. We approved the final version of the manuscript.

Funding. This work was supported by the Russian Foundation for Basic Research (project no. 19-11-00223).

\section{Research Article}

(우(1) The content is published under the terms of the Creative Commons Attribution 4.0 International License (http://creativecommons.org/licenses/by/4.0/)

Please cite this article in press as:

$\mathrm{Kh}$ achatryan Kh. A., Petrosyan H. S. On alternating and bounded solutions of one class of integral equations on the entire axis with monotonic nonlinearity, Vestn. Samar. Gos. Tekhn. Univ., Ser. Fiz.-Mat. Nauki [J. Samara State Tech. Univ., Ser. Phys. Math. Sci.], 2020, vol. 24, no. 4, pp. 644-662. https://doi.org/10.14498/vsgtu1790 (In Russian).

Authors' Details:

Khachatur A. Khachatryan (1) https://orcid.org/0000-0002-4835-943X

D.Sc. (Phys. \& Math. Sci.), Professor; e-mail: khach82@rambler.ru

Haykanush S. Petrosyan (D) https://orcid.org/0000-0002-7172-4730

Cand. Phys. \& Math. Sci., Associate Professor; e-mail: haykuhi25@mail.ru 


\section{References}

1. Kogan M. N. Rarefied Gas Dynamics. New York, Springer Science, 1969, xi+515 pp. https://doi.org/10.1007/978-1-4899-6381-9.

2. Engibaryan N. B., Khachatryan A. Kh. Some convolution-type integral equations in kinetic theory, Comput. Math. Math. Phys., 1998, vol. 38, no. 3, pp. 452-467.

3. Khachatryan Kh. A. Solvability of a conservative integral equation on the half-line, Izv. NAN Armenii. Matematika, 2002, vol. 37, no. 4, pp. 73-80 (In Russian).

4. Khachatryan Kh. A., Sisakyan A. A. On solvability of one class of nonlinear integral equations on whole line, Vestn. of Russian-Armenian (Slavonic) Univ., 2017, no. 2, pp. 25-40 (In Russian).

5. Khachatryan A. Kh., Khachatryan Kh. A. A nonlinear integral equation of Hammerstein type with a noncompact operator, Sb. Math., 2010, vol.201, no.4, pp. 595-606. https:// doi.org/10.1070/SM2010v201n04ABEH004083.

6. Khachatryan Kh. A., Grigoryan S. A. On nontrivial solvability of a nonlinear HammersteinVolterra type integral equation, Vladikavkaz. Mat. Zh., 2012, vol.14, no.2, pp. 57-66 (In Russian).

7. Kolmogorov A. N., Fomin V. S. Elementy teorii funktsii i funktsional'nogo analiza [Elements of the theory of functions and functional analysis]. Moscow, Nauka, 1976, 543 pp. (In Russian)

8. Arabadzhyan L. G., Khachatryan A. S. A class of integral equations of convolution type, Sb. Math., 2007, vol.198, no.7, pp. 949-966. https://doi.org/10.1070/ SM2007v198n07ABEH003868. 